\title{
Mesure de la durée d'émission $X$ de différents éléments irradiés par un laser de puissance subpicoseconde
}

\author{
C. Chenais-Popovics, F. Dorchies ${ }^{1}$, P. Audebert, V. Nagels, J.P. Geindre, \\ J.C. Gauthier, O. Peyrusse ${ }^{2}$, S. Gary ${ }^{2}$ et F. Girard ${ }^{2}$ \\ Laboratoire pour l'Utilisation des Lasers Intenses, UMR 7605 du CNRS, CEA, \\ École Polytechnique, Université Paris VI, 91128 Palaiseau, France \\ ${ }^{1}$ CELIA, Université Bordeaux I, 33405 Talence, France \\ ${ }^{2}$ Commissariat à l'Énergie Atomique, 91680 Bruyères-le-Châtel cedex, France
}

\begin{abstract}
Résumé : Les sources X ultra-brèves créées par laser sont toujours l'objet de recherches fondamentales. La mesure directe de leur durée est maintenant possible avec l'utilisation de caméras à balayage de fente de résolution sub-picoseconde couplées à un cristal de Bragg, apportant une connaissance plus approfondie de la source, tant au niveau de sa durée que de ses caractéristiques spectrales et spatiales. En focalisant un laser 100 $\mathrm{TW}, 300 \mathrm{fs}$, sur divers éléments (Al, Se, Sm), nous avons mesuré la durée de l'émission X dans la gamme $0.7-0.8 \mathrm{~nm}$. L'émission X dure moins de $10 \mathrm{ps}$, pour les trois éléments étudiés.
\end{abstract}

\section{INTRODUCTION}

L'essor des lasers de haute énergie en impulsions ultra-courtes a permis le développement de sources $\mathrm{X}$ brillantes dans le domaine temporel picoseconde ou sub-picoseconde [1]. Au plan de la brillance, ces sources ont des applications potentielles pour le diagnostic des plasmas de fusion, pour l'imagerie médicale (angiographie et mammographie), et la lithographie X-UV. Au plan temporel, elles ouvrent la voie de l'analyse structurale des matériaux avec une résolution temporelle comparable à celle des mécanismes vibrationnels élémentaires [2]. Les mesures temporelles effectuées jusqu'à ce jour sont peu nombreuses car les caméras à balayage de fente ultra-rapides ont été développées relativement récemment $[3,4,5]$. Ces mesures ont été effectuées dans des conditions très variables, tant sur le plan de de la gamme spectrale explorée que des conditions laser (énergie, cadence, et niveau de préimpulsion). Avec les lasers de haute énergie qui ont un taux de répétition faible, l'émission X est enregistrée en un seul tir. Par contre, avec les lasers à haute cadence, la mesure de la durée d'impulsion $X$ est intégrée sur plusieurs tirs et doit être réalisée avec une caméra à balayage de fente subpicoseconde à faible gigue [6]. L'aluminium [7,8] et le tantale [9] ont été les premiers éléments étudiés avec une caméra à balayage. Une durée sub-picoseconde de l'émission $\mathrm{K} \alpha$ du silicium a été mesurée indirectement dans une expérience pompe-sonde [10]. A plus haut flux, les raies $\mathrm{K} \alpha$ de l'aluminium et du vanadium ont été mesurées avec une caméra à balayage de fente autour de 2-3 ps [11].

Plusieurs mécanismes d'émission X sont en jeu dans l'interaction d'un laser sub-picoseconde avec une cible solide ou gazeuse. D'une part, le chauffage d'une très fine couche de plasma à des températures de plusieurs centaines d'eV provoque une émission «thermique », dont la durée est liée à l'évolution hydrodynamique, à la conduction thermique et à la recombinaison du plasma. D'autre part, des électrons suprathermiques interagissent avec la matière et créent des lacunes en couche interne des ions 
ou atomes. Cela produit un rayonnement $\mathrm{K} \alpha$ pendant l'impulsion laser, dont la durée est théoriquement inférieure à la picoseconde [12].

Nous présentons ici quelques mesures récentes de durée d'impulsions $\mathrm{X}$, effectuées avec une caméra à balayage de résolution sub-picoseconde couplée à un cristal de Bragg conique dans ces conditions bien définies, en minimisant le niveau de préimpulsion et le nombre d'électrons supra-thermiques.

\section{MONTAGE EXPÉRIMENTAL}

L'installation laser néodyme $100 \mathrm{TW}$ du LULI a été utilisée pour produire des sources $\mathrm{X}$ sur des cibles solides d'éléments de numéros atomiques très différents : l'aluminium $(\mathrm{Z}=13)$, le sélénium $(\mathrm{Z}=34)$ et le samarium $(Z=62)$. Des impulsions doublées en fréquence $(\lambda=0.53 \mu \mathrm{m})$, de durée $300 \mathrm{fs}$ et d'intensité 1 à 10 Joule étaient focalisées avec une parabole hors axe de focale $750 \mathrm{~mm}$. A la meilleure focalisation $\left(20 \times 40 \mu \mathrm{m}^{2}\right)$, l'intensité laser sur cible atteignait $710^{18} \mathrm{~W} / \mathrm{cm}^{2}$. L'émission était résolue temporellement et spectralement en couplant un cristal de Bragg conique à une caméra à balayage de fente, permettant de mesurer la gamme spectrale autour de $1600 \mathrm{eV}$. La géométrie conique du cristal est analogue à la géométrie focalisante Van Hamos, mais elle permet de focaliser le spectre dans le plan de la photocathode, perpendiculaire à l'axe plasma - détecteur. Nous avons choisi la région spectrale qui couvre la raie de résonance $1 \mathrm{~s}^{2}-1 \mathrm{~s} 2 \mathrm{p}$ de l'aluminium héliumoüde, ses satellites lithiumoïdes ainsi que les transitions $\mathrm{K} \alpha$ (transitions en couche interne $\mathrm{n}=2 \Rightarrow \mathrm{n}=1$ ) des ions $\mathrm{Al}^{6+}$ à $\mathrm{Al}^{11+}$. On peut observer ces transitions en émission, ou en absorption en utilisant comme source $\mathrm{X}$ annexe un plasma de samarium. Les transitions $3 \mathrm{~d}-4 \mathrm{f}$ du samarium ionisé de 32 à 40 fois sont dans ce domaine de longueur d'onde. Cette région recouvre aussi les transitions $2 \mathrm{p}-3 \mathrm{~d}$ des ions sélénium isoélectroniques du néon.

\section{CARACTÉRISATION DE LA CAMÉRA À BALAYAGE DE FENTE}

Avant d'effectuer des mesures de durée d'impulsions X, nous avons défini les conditions de bon fonctionnement de la caméra à balayage de fente. Nous avons mesuré la vitesse de balayage de la caméra en retardant une partie du faisceau avec une lame de verre d'épaisseur $1 \mathrm{~cm}$. Nous avons mesuré une vitesse de $200 \pm 10 \mathrm{fs} / \mathrm{pixel}$, soit $5.3 \pm 0.2 \mathrm{ps} / \mathrm{mm}$. Avec le générateur de rampe de tension (pulseur) utilisé au LULI, nous avons obtenu une gigue d'environ $10 \mathrm{ps,} \mathrm{qui} \mathrm{est} \mathrm{fortement} \mathrm{réduite} \mathrm{par}$ l'utilisation d'un pulseur à interrupteur d'Auston [6]. En mode accumulation (jusqu'à 600000 tirs), la gigue, mesurée au laboratoire CELIA, était suffisamment faible pour permettre une mesure avec une résolution de $1.2 \mathrm{ps}$.

Nous avons aussi défini l'intensité de saturation de l'optique électronique. En effet, cette caméra a une dynamique faible, et il est important de définir dans quelles conditions on effectue des mesures correctes de durée d'impulsion. Pour cela, nous avons utilisé des filtres de mylar d'épaisseur $6 \mu \mathrm{m}$, montés sur un support motorisé permettant de faire varier de 0 à 3 le nombre de feuilles de mylar, donnant un facteur d'atténuation entre 0 et 10 . Par ailleurs, nous avons aussi utilisé un filtre à échelon comportant 0,1 et 2 feuilles de mylar de même épaisseur. La figure 1 illustre l'effet de saturation de la 
caméra. La figure la montre un spectre d'aluminium, sur lequel les intensités dans le continuum, dans les raies satellites et dans la raie He $\alpha$ sont très différentes. On voit que l'émission des raies parait démarrer avant le continuum, ce qui est purement dû à un effet de saturation. En utilisant les différents filtres de mylar (Fig. 1b), nous avons défini le niveau de sortie de la caméra permettant d'éviter la saturation. Cette image a été obtenue en irradiant une cible de samarium avec une intensité de 10 Joule. La partie non filtrée est saturée et démarre très tôt. La partie centrale est à peine saturée, et la partie gauche n'est pas saturée. Ceci nous a permis de définir que au dessus d'un nombre de 75 coups au dessus du bruit de fond reçus par la caméra CCD, la saturation se fait sentir. La dynamique de la caméra est donc extrêmement faible. Pour exploiter des spectres de grande dynamique, il faut effectuer plusieurs tirs lasers en filtrant différemment le spectre, la mesure étant alors sujette aux fluctuations tir à tir des résultats.

(a)

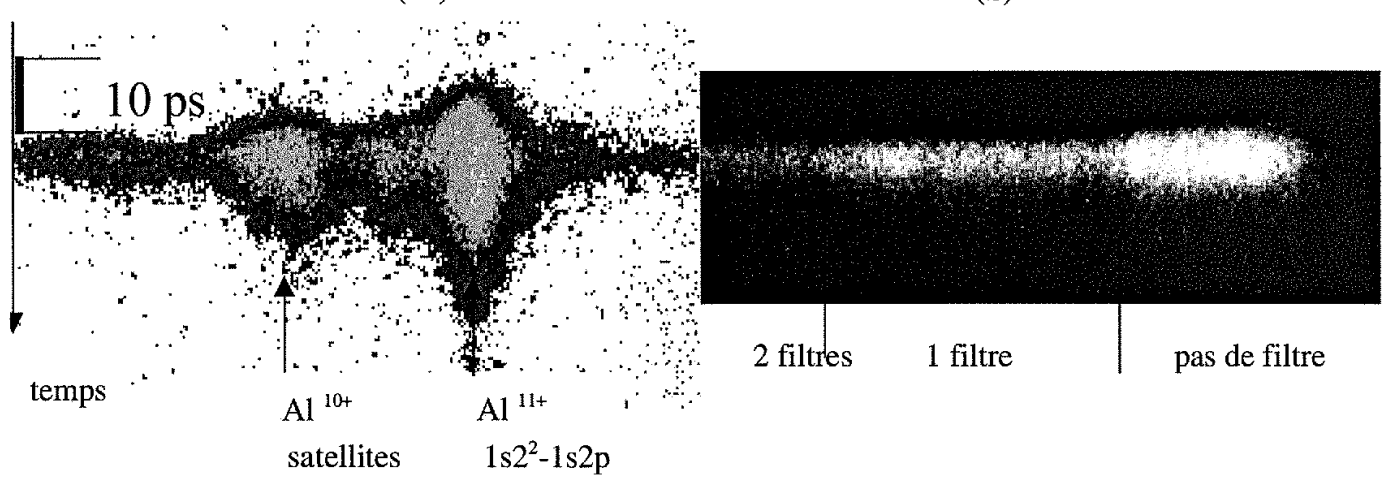

Figure 1: Spectre de l'aluminium (a) et du samarium (b) montrant la saturation de la caméra à balayage. Le filtre à échelon utilisé pour le samarium comporte de 0 à 2 feuilles de mylar d'épaisseur $6 \mu \mathrm{m}$.

\section{MESURE DE LA DURÉE D'IMPULSIONS X ÉMISES PAR DIFFÉRENTS ÉLÉMENTS}

Nous avons mesuré les spectres d'aluminium, de sélénium et de samarium dans la gamme spectrale $0.7-0.8 \mathrm{~nm}$, en fonction du temps, pour une énergie laser de 1 et 10 Joule. Les spectres de samarium sont parfaitement continus bien que la région spectrale soit centrée sur les raies $3 \mathrm{~d}-4 \mathrm{f} d u$ samarium. En régime nanoseconde, des raies apparaissent dans la gamme spectrale étudiée, mais en régime femtoseconde, les conditions de densité et température font disparaître ces raies. Nous avions déjà vu cet effet sur des mesures intégrées temporellement pour lesquelles la source de samarium était utilisée pour sonder un plasma d'aluminium par absorption [14]. La durée de la source de samarium est reportée sur la figure 2. La mesure donnée par le tracé est de 2.6 et 4.2 ps respectivement, pour une énergie laser de 1 et 10 Joule, soit une intensité de $710^{17}$ et $710^{18} \mathrm{~W} / \mathrm{cm}^{2}$. 


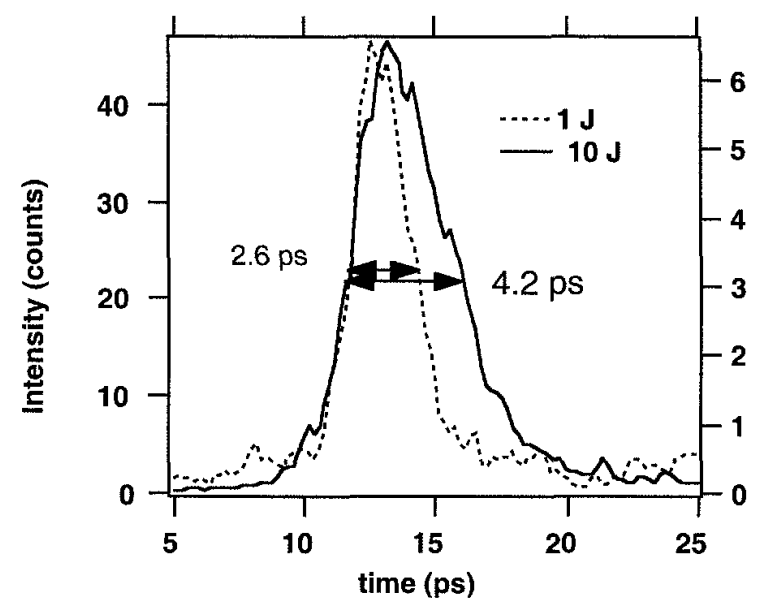

Figure 2 : Durée d'émission du samarium pour deux valeurs de l'énergie laser (1 et $10 \mathrm{~J}$ )
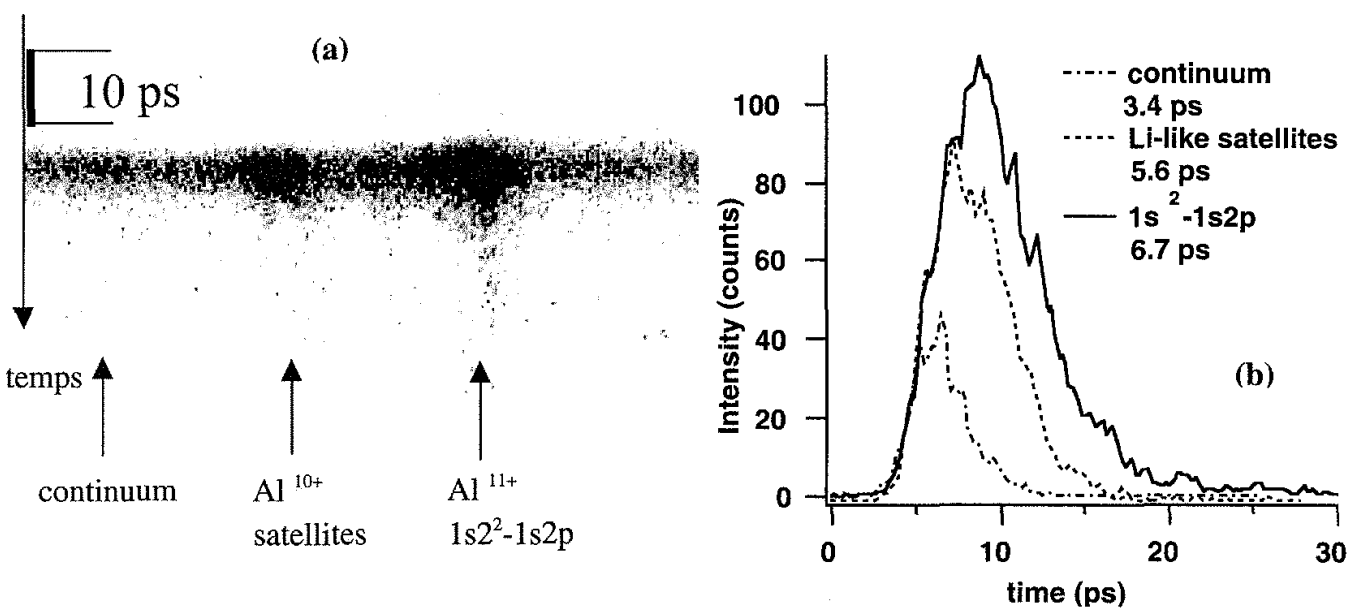

Figure 3 : Spectre de l'aluminium en fonction du temps. Image brute (a) et durée d'impulsion (b) mesurées pour la raie de résonance, les satellites lithiumoïdes et le continuum.

La figure 3 montre les résultats obtenus avec l'aluminium, pour une intensité laser de $3 \times 10^{17} \mathrm{~W} / \mathrm{cm}^{2}$. L'image obtenue (Fig. 3a) montre la raie de résonance héliumoïde $1 s^{2}-1 s 2 p$, ses satellites lithiumoïdes entourés par du continuum. La raie de résonance a une durée à mi-hauteur de $6.7 \mathrm{ps.} \mathrm{Un} \mathrm{pied} \mathrm{assez} \mathrm{long}$ suit cette émission, pendant la phase de recombinaison du plasma. Les satellites sont plus courts (5.6 ps à mi-hauteur), et n'émettent pas pendant la recombinaison du plasma. Enfin, le continuum est encore plus court et dure $3.4 \mathrm{ps}$, durée similaire à ce que l'on obtient avec le samarium. 
Avec le sélénium, les durées sont comparables, les raies émettant plus longtemps que le continuum. Pour une intensité laser de $10^{18} \mathrm{~W} / \mathrm{cm}^{2}$, le continuum dure $3.3 \mathrm{ps}$, et les raies $2 \mathrm{p}$ - $3 \mathrm{~d}$ durent 3.3 et $4.6 \mathrm{ps}$. A plus haute intensité $\left(710^{18} \mathrm{~W} / \mathrm{cm}^{2}\right)$, nous mesurons 6 ps pour le continuum, et 7.2 et $7.3 \mathrm{ps}$ pour les deux raies $2 p-3 d$. La durée de la raie ${ }^{\prime} P_{1}$ est toujours légèrement plus courte que celle de la raie ${ }^{3} \mathrm{D}_{1}$. La compréhension de cette différence demandera un travail complémentaire de simulation, qui sera réalisé en couplant le code de physique atomique AVEROES/TRANSPEC [15] à une simulation hydrodynamique de l'évolution du plasma.

La durée d'émission de plasmas créés sur cible solide sont très proches de celles obtenues sur un jet d'agrégats. Une caméra du même type a été utilisée au CELIA, en mode répétitif, avec une résolution de 1.2 ps. La durée de l'émission $X$, autour de $3 \mathrm{KeV}$, d'agrégats d'argon (rayon $30 \mathrm{~nm}$ ), irradiés avec une faisceau d'intensité $5 \times 10^{15} \mathrm{~W} / \mathrm{cm}^{2}$, de durée 700 fs a été mesurée à $3.5 \pm 0.5 \mathrm{ps}$, donc très proche des valeurs obtenues sur cible solide.

\section{CONCLUSION}

Le couplage d'une caméra à balayage de fente sub-picoseconde avec un cristal conique permet de mesurer la durée d'une source $\mathrm{X}$, avec résolution spectrale. La caméra permet d'obtenir une résolution de 500 à 800 fs en mode monocoup. Equipée d'un pulseur à switch d'Auston, la caméra peut fonctionner en mode itératif avec une excellente résolution de $1.2 \mathrm{ps}$.

Les sources $X$ ainsi obtenues ont des durées inférieures à $10 \mathrm{ps}$, quel que soit le matériau irradié par laser. Ceci a un très grand intérêt pour sonder la matière pendant des temps très courts. Les spectres obtenus avec le samarium présentent un spectre plat dans la région des raies d'absorption $\mathrm{K}$ de l'aluminium. Ceci pourra être mis à profit pour utiliser un diagnostic de spectroscopie d'absorption à très haute résolution temporelle, d'interêt pour l'astrophysique et la fusion inertielle. Ceci renforce l'interêt de coupler un laser ultra-court, pétawatt avec un laser de puissance, comme la «Ligne d'Intégration Laser » au CEA Bordeaux, et comme le laser kilojoule du LULI.

\section{REMERCIEMENTS}

Ce travail a été réalisé avec l'aide des équipes technique du LULI, en particulier E. Veuillot, C. Lebris et C. Felix. La synchronisation de la caméra utilise un interrupteur d'Auston réalisé par D. Gonthier. Les cibles ont été réalisées au Lawrence Livermore National Laboratory et au Laboratoire des Cibles de l'Institut de Physique Nucléaire. Ce travail a été financé partiellement par la Comunauté Européenne sous le contrat XPOSE HPRN-CT-2000-00160.

\section{RÉFÉRENCES}

[1] Pour une revue récente, voir les contributions dans: Les nouvelles sources ultra-brèves de rayons $X$ et leurs applications, C.R.A.S. IV, Tome 1, (2000) Ed: J.-C. Gauthier.

[2] A. Rousse, C. Rischel, et J.-C. Gauthier, Review Modern Physics 73, 17-31 (2001) 
[3] M.M. Murmane, H.C. Kapteyn et R. Falcone, Appl. Phys. Lett. 56 (1990) 1948 -1950

[4] A. Mens, R. Sauneuf, D. Schirmann, R. Verrecchia, P. Audebert, J.C. Gauthier, J.P. Geindre, A. Antonetti, J.P. Chambaret, G. Hamoniaux et A. Mysyrowicz, Proc. SPIE 1757 (1993) 8-18, Ultrahigh- and High-Speed Photography, Videography, and Photonics

[5] P. Gallant, P. Forget, F. Dorchies, Z. Jiang, J. C. Kieffer, P. A. Jaanimagi, J. C. Rebuffie,

C. Goulmy, J. F. Pelletier and M. Sutton, Rev. Sci. Instrum. 71 (2000) 3627-3633

[6] C. Belzile, J. C. Kieffer, C. Y. Côté, T. Oksenhendler et D. Kaplan, Rev. Sci. Instrum. 73 (2002) 1617-1620

[7] R. Shepherd, R. Booth, B. Young, D. Price, R. More, R.W. Lee, R.E. Stewart, Proc. SPIE 3157, (1997) 2-9, Applications of X Rays Generated from Lasers and Other Bright Sources.

[8] U. Andiel, K. Eidmann et K. Witte, Phys. Rev. E 63 (2001) 026407-1 à 7

[9] J.F. Pelletier, M. Chaker et J.C. Kieffer, Appl. Phys. Lett. 69 (1996) 2172-2174

[10] C. Rischel, A. Rousse, I. Uschmann, P. A. Albouy, J.P. Geindre, P. Audebert, J.C. Gauthier, E. Förster, J.L. Martin, A. Antonetti, Nature, 390 (1997) 490-492

[11] J.C. Kieffer, Z. Jiang, A. Ikhlef et C.Y Côté, J. Opt. Soc. Am. B 13 (1996) 132-137

[12] T. Feurer, A. Morak, I. Uschmann, Ch. Ziener, H. Schwoerer, Ch. Reich, P. Gibbon, E. Förster, R. Sauerbrey, K. Ortner et C.R. Becker, Phys. Rev. E 65 (2001) 016412

[13] R.S. Marjoribanks, M.C. Richardson, P.R. Audebert, D.K. Bradley, G.G. Gregory et P.A. Jaanimagi, , Proc. SPIE 831 (1987) 185-198, X-rays from Laser Plasmas

[14] M. Fajardo, P. Audebert, P. Renaudin, H. Yashiro, R. Shepherd, J.C. Gauthier et C. ChenaisPopovics, Phys. Rev. Letters 86 (2001) 1231-1234

[15] O. Peyrusse, J. Phys. B 33 (2000) 4303-4321 